\title{
Growth and Development
}

\author{
Marvin L. Birnbaum, MD, PhD
}

Some people will never learn anything, for this reason, because they understand everything too soon.

Pope, Thoughts on Various Subjects

The trouble with our times is that the future is not what it used to be.

Paul Valéry

An examination of both the quantity and quality of the abstracts for the 14th World Congress on Disaster and Emergency Medicine (14th WCDEM) published in the previous issue of PDM (20:2) and augmented by those in this issue reflects how far disaster, prehospital, and emergency medicine have come in so short a period of time. This growth has been steady since the 9th WCDEM in 1995 (Figure 1). Our discipline has matured and is becoming a better science. Not only has the number of submitted abstracts increased, but also more notably, the number of authors is increasing at an even faster rate. More people are developing an interest in our science. We are coming of age!

What is contributing to this growth and development, and what must we do with our rapidly expanding wealth of information? Unfortunately, the number and severity of events that could result in a disaster continue to increase. ${ }^{1}$ The numbers of persons affected by these events and the disasters that often follow have become astronomical with the total number affected by disasters involving the majority of the world's population. Along with this unfortunate circumstance, the number of medical responses also is growing, and hence, so is our experience level. Although the number of events is increasing, in general terms, the total number of deaths related to these events is decreasing - a tribute to our ability to become prepared and our enhanced ability to respond. ${ }^{1}$ However, it is not just the numbers that are changing; our knowledge of what we are doing - and the public's awareness of what we are doing-is increasing. This was manifest by the unprecedented outpouring of resources for assistance to those countries ravaged by the recent events in the Indian Ocean.

In order to obtain the resources that we need to become even better at what we do, we must keep the world's attention on our activities. So far, very little of supposedly available resources have come to disaster and emergency medicine. In order for our growth and development to continue, we need resources directed our way.
The growth and rapid development of our specialty is a testament to the increasing recognition of the importance of Disaster and Emergency Medicine in the world of medicine. As one examines the backgrounds and disciplines of the presenters at the 14th WCDEM, one must be impressed by the huge breadth of information that is encompassed by Disaster and Emergency Medicine-our science interfaces and is interdependent with so many of the other basic societal functionsmore so than for any other element of medicine. We cannot operate within a vacuum. Our discipline spans borders and cultures; disasters have no boundaries. Disasters are a world problem and do not occur only in the country, which was the unfortunate victim of a disaster. Our growth and development represent an increasing interest in defining what we do and how we do it. Our science is wide open and hungry, and currently, has an unprecedented importance for the future of our planet and the people who inhabit it.

What must we do to improve and make the increasing quantity of collected information more useful? Currently, we are unable to amalgamate all of the data we are acquiring into meaningful information that can be applied to helping us do our jobs even better. The lack of uniformity in presentation structure and definitions of the terms used are major barriers to the value, as well as to the application, of the findings. To be useful, the information must be made available to us with little effort and in such a manner as to be of maximum value in helping us shape what we can, should, and should not do.

The next and logical step must be to mandate that all of the data and information presented at any WADEM Congress follow the Templates and Guidelines provided in "Health Disaster Management: Guidelines for Research and Evaluation in the Utstein Style". ${ }^{1}$ At this Congress in Edinburgh, I challenge the chairs and moderators of each of the sessions to do just this: summarize the findings presented in their sessions using the terminology and structure of the Guidelines. These summaries should: (1) form the basis of the conclusions reached by the Congress; (2) incorporate the expertise of the moderators and chairs; and (3) be worthy of publication in this Journal. We must begin to structure all of our disaster-related research and evaluations (including the descriptions of the events, the damage created, and the disturbances to the healthcare system) to conform to the 


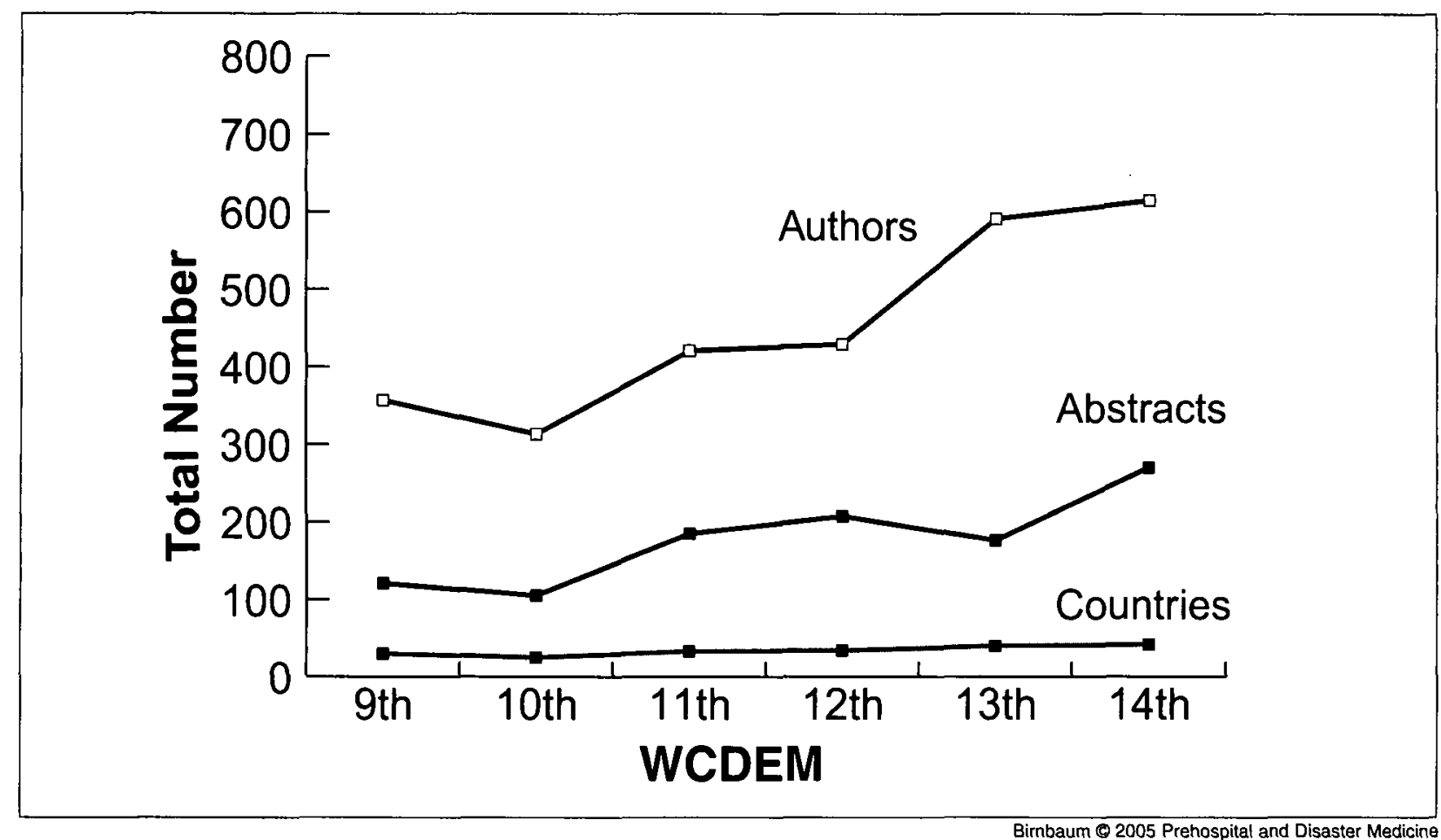

Figure 1-Numbers of authors, abstracts, and countries with abstracts accepted to be part of the World Congress for Disaster and Emergency Medicine (WCDEM) for 1995-2005.

conceptual and operational structures of the Guidelines. Without so doing, we will not be able to glean the information from the data we already have accumulated. We dare not lose what we already have. The way qualitative data gains meaning and achieves internal (cause:effect) validity, is by repeatedly demonstrating the same phenomena. It behooves WADEM and this Journal to assure that you have ready access to this material in a manner that is useful in meeting your specific needs. And WADEM must convince other agencies of the utility of the structures provided and to assist in the dissemination of these structures. Until appropriate education and training in the use of the Guidelines are provided, it is unrealistic to believe that these structures will come into common use. PDM will do all it can to facilitate this process.

The abstracts as well as the Programme content for the 14th WCDEM are a testimony to the breadth and depth of what Disaster, Emergency, Prehospital, and Humanitarian Medicine is all about. The scientific papers that will be presented at the Congress are interspersed throughout 17 themes. Moreover, the abstracts have been submitted from authors from 42 countries, attesting to the truly global nature of this 14 th WCDEM. More and more of you are responding to the increasing number of crises in this world, and are attempting to define the actual needs of the affected population and the effectiveness of the responses in meeting these needs. We are beginning to get a grasp on the pathophysiology of disasters. We are becoming increasingly aware and critical of what we do. The evaluations of the responses to the tsunamis that arose from the Indian Ocean in December will comprise several sessions of the Congress. Much can be learned from the evaluation of these responses, many of which still are ongoing. There is much to learn for all of us at this Congress.

Appropriately, the themes of the Congress are concordant with the Task Forces and Committees of the WADEM. The WADEM has defined the important areas that are open for discussion and research. The output from the task forces will determine the direction of the WADEM. Membership in the task forces is open to all members; it is up to you to determine how and what WADEM will accomplish during the next decade.

I congratulate the members of the Organizing Committee of the 14th WCDEM for their vision and ability to stimulate the scientists to contribute to the Congress. And as you participate in what I believe will be the best, most comprehensive World Congress on Disaster and Emergency Medicine ever convened, I urge you to think about how the information to which you are exposed could be of greater value to you. Find me during the Congress, and share your thoughts. I am looking forward to talking with you in Edinburgh. Have a safe trip.

The only use of a knowledge of the past is to equip us for the present. No more deadly harm can be done to young minds than by deprecation of the present. The present contains all that there is. It is holy ground; for it is the past, and it is the future.

Alfred North Whitehead

\section{Reference}

1. Task Force on Quality Control in Disaster Medicine/World Association for Disaster and Emergency Medicine: Health Disaster Management: Guidelines for Evaluation and Research in the "Utstein Sytle." Prehosp Disast Med 2002;17(Suppl 3). 